\title{
ITERATIVE MULTISCALING STRATEGY INCORPORATED INTO TIME DOMAIN INVERSE SCATTERING METHOD FOR CROSS-BOREHOLE IMAGING
}

\author{
Toshifumi Moriyama ${ }^{\dagger}$, Giacomo Oliveri $^{\ddagger}$, Andrea Massa ${ }^{\ddagger}$, and Takashi Takenaka ${ }^{\dagger}$ \\ ${ }^{\dagger}$ Department of Electrical and Electronic Engineering, Nagasaki University \\ Nagasaki 852-8521, JAPAN, E-mail: takenaka@nagasaki-u.ac.jp \\ ${ }^{\ddagger}$ ELEDIA Group - Department of Information Engineering and Computer Science, \\ University of Trento, Via Sommarive 14, 1-38050 Trento, ITALY
}

\begin{abstract}
We consider cross-borehole imaging of buried objects embedded in a large search area by using a time domain inverse analysis. For simplicity, a two-dimensional model is examined. In order to avoid trap into false solution and enhance the achievable spatial resolution, the iterative multiscaling strategy combined with the forward-backward time-stepping method is proposed. Preliminary results show the effectiveness of proposed method.
\end{abstract}

Index Terms - inverse scattering, iterative multiscaling, microwave imaging, cross-borehole, time domain

\section{INTRODUCTION}

Cross-borehole radar can access objects buried tens of meters underground and image geophysical structure between two boreholes [1-3]. Cross-borehole imaging often faces a problem of detecting high-contrast buried objects in a large search region compared to the operating wavelength. In such a case, tomographic imaging techniques based on inverse scattering analysis often tap into false solution (i.e., a wrong scatterers retrieval) when a high-resolution inversion is carried out or yield smooth scatterer reconstructions, otherwise. To overcome these drawbacks, a novel tomographic borehole imaging technique is presented in this paper. More in detail, the detection of unknown objects in wide search regions with a sufficient resolution is yielded by integrating the forward-backward time-stepping (FBTS) algorithm $[4,5]$ with the iterative multiscale scheme (IMS) [6-8].

\section{FORMULATION}

Let us consider a two-dimensional borehole radar imaging problem. As shown in Fig. 1, the investigation domain $D$ is successively irradiated by a microwave pulse generated at transmitter points $\mathbf{r}=\mathbf{r}_{m}^{t} \quad(m=1,2, \cdots, M)$ with an

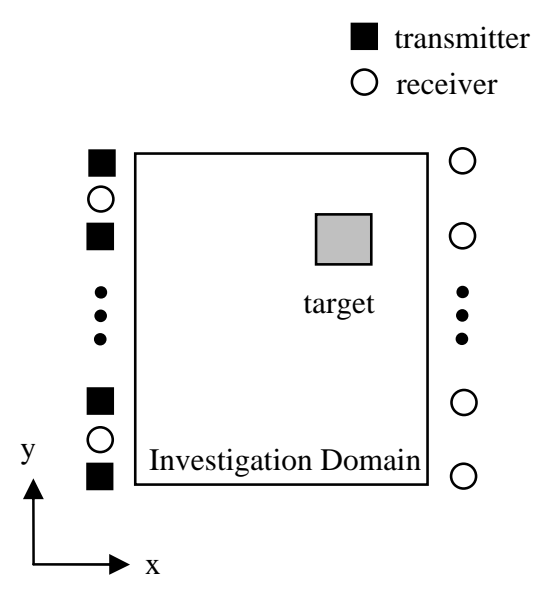

Fig. 1. Geometry of the problem

electric current $\mathbf{J}_{m}(\mathbf{r}, t)$ in the left borehole. The pulse is scattered by the unknown object belonging to the scatterer's investigation domain and the total fields are collected at receiver points $\mathbf{r}=\mathbf{r}_{n}^{r}(n=1,2, \cdots, M)$ in both left and right boreholes. For the sake of simplicity, two-dimensional TM case is considered (i.e., the scatterers are cylindrical objects with axes along the z-direction and the current sources are infinitely long line currents along the same direction). Moreover, the objects are also assumed as lossless and nonmagnetic material and are embedded in an uniform isotropic background with constant permittivity $\varepsilon_{b}$. The total fields $\mathbf{v}_{m}$ satisfy Maxwell's equations:

$$
L \mathbf{v}_{m}=\mathbf{J}_{m}
$$

where

$$
\mathbf{v}_{m}(\mathbf{r}, t)=\left[\begin{array}{c}
E_{z, m}(\mathbf{r}, t) \\
\eta H_{x, m}(\mathbf{r}, t) \\
\eta H_{y, m}(\mathbf{r}, t)
\end{array}\right], \quad \mathbf{J}_{m}(\mathbf{r}, t)=\left[\begin{array}{c}
\eta J_{z, m}(\mathbf{r}, t) \\
0 \\
0
\end{array}\right],
$$




$$
L \equiv A \frac{\partial}{\partial x}+B \frac{\partial}{\partial y}-C \frac{\partial}{\partial(c t)} .
$$

$c$ and $\eta$ being the speed of light and the intrinsic impedance in the background medium, respectively. The matrices in (2) are given by

$$
\begin{aligned}
A & =\left[\begin{array}{lll}
0 & 0 & 1 \\
0 & 0 & 0 \\
1 & 0 & 0
\end{array}\right], \quad B=\left[\begin{array}{ccc}
0 & -1 & 0 \\
-1 & 0 & 0 \\
0 & 0 & 0
\end{array}\right] \\
C & =\left[\begin{array}{ccc}
\varepsilon_{r}(\mathbf{r}) & 0 & 0 \\
0 & 1 & 0 \\
0 & 0 & 1
\end{array}\right]
\end{aligned}
$$

where $\varepsilon_{r}(\mathbf{r})$ is the relative permittivity of the objects. By assuming that the current is turned on at time $t=0$, the initial condition of the generated electromagnetic fields is

$$
\mathbf{v}_{m}(\mathbf{r}, 0)=\mathbf{0} \text {. }
$$

\subsection{Forward-backward Time-stepping Algorithm}

The inverse scattering problem considered here can be formulated as an optimization one: finding the relative permittivity distribution $\varepsilon_{r}(\mathbf{r})$ which minimizes the following cost functional

$$
\begin{aligned}
F\left(\varepsilon_{r}\right) & =\sum_{m=1}^{M} \sum_{n=1}^{N} \int_{0}^{T} K_{m}\left(\mathbf{r}_{n}^{r}, t\right) \times \\
& \left|E_{z, m}\left(\varepsilon_{r}(\mathbf{r}) ; \mathbf{r}_{n}^{r}, t\right)-\tilde{E}_{z, m}\left(\mathbf{r}_{n}^{r}, t\right)\right|^{2} d t
\end{aligned}
$$

where $\tilde{E}_{z, m}\left(\mathbf{r}_{n}^{r}, t\right)$ and $E_{z, m}\left(\varepsilon_{r}(\mathbf{r}) ; \mathbf{r}_{n}^{r}, t\right)$ are the measured and the calculated electric fields at the $n$th receiver point due to the current source $\mathbf{J}_{m}$. The upper limit of integration $T$ is the time duration of the measurement and $K_{m}\left(\mathbf{r}_{n}^{r}, t\right)$ is a nonnegative weighting function equal to zero at $t=T$.

A gradient-based technique (namely the FBTS [4]) is employed to minimize $F\left(\varepsilon_{r}\right)$. More specifically, the Fréchet differential $F^{\prime} \delta \varepsilon_{r}$ of the functional is given by

$$
F^{\prime} \delta \varepsilon_{r}=\left\langle g(\mathbf{r}), \delta \varepsilon_{r}(\mathbf{r})\right\rangle
$$

being

$$
\langle a(\mathbf{r}), b(\mathbf{r})\rangle \equiv \int_{D} a(\mathbf{r}) b(\mathbf{r}) d S .
$$

The gradient $g$ of the functional is given by

$$
\begin{aligned}
g(\mathbf{r})= & 2 \sum_{m=1}^{M} \int_{0}^{T} w_{1, m}\left(\varepsilon_{r}(\mathbf{r}) ; \mathbf{r}, t\right) \\
& \times \frac{\partial}{\partial(c t)} E_{z, m}\left(\varepsilon_{r}(\mathbf{r}) ; \mathbf{r}, t\right) d t
\end{aligned}
$$

where $w_{1, m}$ is the first component of the adjoint field vector $\mathbf{w}_{m}\left(\varepsilon_{r}(\mathbf{r}) ; \mathbf{r}, t\right)$ which is the solution of the following equation

$$
L \mathbf{w}_{m}=\mathbf{u}_{m}
$$

subject to the condition

$$
\mathbf{w}_{m}\left(\varepsilon_{r}(\mathbf{r}) ; \mathbf{r}, T\right)=\mathbf{0} \text {. }
$$

In (9), $\mathbf{u}_{m}$ is the weighted residual (i.e., the weighted difference of the calculated and measured field data) whose first component is equal to

$$
\begin{gathered}
\sum_{n=1}^{N} K_{m}\left(\mathbf{r}_{n}^{r}, t\right)\left[E_{z, m}\left(\varepsilon_{r}(\mathbf{r}) ; \mathbf{r}_{n}^{r}, t\right)\right. \\
\left.-\tilde{E}_{z, m}\left(\mathbf{r}_{n}^{r}, t\right)\right] \delta\left(\mathbf{r}-\mathbf{r}_{n}^{r}\right)
\end{gathered}
$$

while its second and third components are zeros. It is worth nothing that the Maxwell's operator $L$ is self-adjoint in the lossless case.

Let the $k$ th step be referred to by superscript ' $k$ '. The estimate of the relative permittivity $\varepsilon_{r}$ at the $(k+1)$ th iteration is given by the following updating formula:

$$
\varepsilon_{r}^{k+1}(\mathbf{r})=\varepsilon_{r}^{k}(\mathbf{r})+\alpha^{k} d^{k}(\mathbf{r})
$$

where $d^{k}$ is a search direction, which in this paper is chosen to be the Polak-Ribière-Polyak conjugate direction,

$$
\begin{aligned}
d^{k}(\mathbf{r})= & -g^{k}(\mathbf{r}) \\
& +\frac{\left\langle g^{k}(\mathbf{r})-g^{k-1}(\mathbf{r}), g^{k}(\mathbf{r})\right\rangle}{\left\langle g^{k-1}(\mathbf{r}), g^{k-1}(\mathbf{r})\right\rangle} d^{k-1}(\mathbf{r})
\end{aligned}
$$

The step size $\alpha^{k}$ is determined approximately by linearizing $E_{z, m}\left(\varepsilon_{r}(\mathbf{r}) ; \mathbf{r}_{n}^{r}, t\right)$ with respect to $\alpha^{k}$.

Let $L$ be the side of the square region of interest $D$. Taking into account of the limited bandwidth properties of the measured scattering data, the unknown relative permittivity $\varepsilon_{r}(x, y)$ is expanded into 2-D truncated

Fourier series over the domain $D^{s}$ as in the reference [9]:

$$
\varepsilon_{r}(x, y)=\sum_{p=-P}^{P} \sum_{q=-Q}^{Q} A_{p, q} \exp \left[j \frac{2 \pi}{L}(p x+q y)\right] \text {. }
$$

Then, the functional $F\left(\varepsilon_{r}\right)$ reduces to a function of the coefficients $A_{p, q}(-P \leq p \leq P ;-Q \leq q \leq Q)$. The differential $\delta \varepsilon_{r}$ is represented as 


$$
\delta \varepsilon_{r}(x, y)=\sum_{p=-P}^{P} \sum_{q=-Q}^{Q} \delta A_{p, q} \exp \left[j \frac{2 \pi}{L}(p x+q y)\right] .
$$

Substituting (15) into (6), we get the differential of the function $F\left(\left\{A_{p, q}\right\}\right)$ with respect to the coefficients $A_{p, q}$ :

$$
F^{\prime} \delta \varepsilon_{r}=(G, \delta A)
$$

where $G$ and $A$ are $(2 P+1) \times(2 Q+1)$ matrices whose pqth entries are $\delta A_{p, q}$ and $G_{p, q}$ given by

$$
\begin{aligned}
G_{p, q} & =\int_{D} g(x, y) \exp \left[-j \frac{2 \pi}{L}(p x+q y)\right] d x d y, \\
& -P \leq p \leq P ;-Q \leq q \leq Q
\end{aligned}
$$

and the inner product $(A, B)$ is defined by

$$
(A, B)=\sum_{p=-P}^{P} \sum_{q=-Q}^{Q} A_{p, q}^{*} B_{p, q} .
$$

The updating formula for the coefficients $A_{p, q}$ at the $(k+1)$ th iteration is given by

$$
A_{p, q}^{k+1}=A_{p, q}^{k}+\alpha^{k} D_{p, q}^{k}
$$

where $D_{p, q}^{k}$ is a search direction for the coefficients and is represented by

$$
D_{p, q}^{k}=-G_{p, q}^{k}+\frac{\left(G^{k}-G^{k-1}, G^{k}\right)}{\left(G^{k-1}, G^{k-1}\right)} D_{p, q}^{k-1} .
$$

\subsection{Iterative Multiscaling Algorithm}

The reconstruction using a simple optimization technique in a large investigation domain with limited-view measurements such as borehole imaging often fails trapped into a false solution. On way to tackle this problem is to reduce as much as possible the extension of the domain of investigation enclosing the unknown objects. In this respect, we adopt an iterative procedure based on a multiresolution approach [6]. First, a 'coarse' reconstruction of the unknown dielectric profile is searched for in the whole investigation domain. Then, the investigation domain is reduced iteratively to 'zoom' the region to which the object belongs and reconstruct the dielectric profile more accurately with higher resolution. The multistep procedure is stopped when the reduction of the investigation domain becomes smaller than a prescribed value. In more detail, at each step of the iterative process, by means of the acquired a priori information (achieved at the previous steps), the scatterer is localized and a zoomed square investigation domain $D_{(s)}$ is centered at

$$
\begin{gathered}
x_{(s)}=\frac{\int_{D_{(s-1)}} x\left|\Delta \varepsilon_{r}(x, y)\right| d x d y}{\int_{D_{(s-1)}}\left|\Delta \varepsilon_{r}(x, y)\right| d x d y}, \\
y_{(s)}=\frac{\int_{D_{(s-1)}} y|\Delta \varepsilon(x, y)| d x d y}{\int_{D_{(s-1)}}|\Delta \varepsilon(x, y)| d x d y} .
\end{gathered}
$$

where $\Delta \varepsilon_{r} \equiv \varepsilon_{r}-\varepsilon_{r b}$ and $\varepsilon_{r b}$ is the relative permittivity of the background medium. The side $L_{(s)}$ of the investigation domain $D_{(s)}$ is defined as

$$
L_{(s)}=2 \sqrt{\frac{\int_{L_{(s-1)}} \Delta r(x, y) \Delta \varepsilon(x, y) d x d y}{\int_{L_{(s-1)}} \Delta \varepsilon(x, y) d x d y}}
$$

where $\Delta r(x, y) \equiv\left(x-x_{(s)}\right)^{2}+\left(y-y_{(s)}\right)^{2}$.

The multistep procedure is terminated when the 'stationary condition' on the reconstruction is satisfied [8].

\section{NUMERICAL RESULTS}

In this section, some preliminary results are reported to assess the effectiveness of the proposed IMS-FBTS method. Let us consider a tunnel-like object filled with air $\left(\varepsilon_{r}=1\right)$ embedded in a lossless background medium with $\varepsilon_{b, r}=5$. The object is a square with side $a=1.0 \mathrm{~m}$. The distance between the left and right borehole is $22.8 \mathrm{~m}$. Eight transmitter positions are uniformly distributed in the left borehole at a 3.3-m interval, i.e., 23.1-m source domain. Fifteen receiver positions are placed along 23.1-m measurement domain in each borehole. The FDTD solution space is a square area of $26.4 \times 26.4 \mathrm{~m}^{2}$ wide, which is partitioned into $176 \times 176$ cells of size $\Delta x=\Delta y=0.15 \mathrm{~m}$ and time step size $\Delta t=0.35 \mathrm{~ns}$. A fifteen-cell perfectly matched layer is used as an absorbing boundary condition for the FDTD grid. The time variation of the current source is given by

$$
I(t)=e^{-\left[\left(t-t_{0}\right) / 0.29 T_{s}\right]^{2}} \sin \left(2 \pi f_{c} t\right)
$$

where $t_{0}=48.45 \mathrm{~ns}, T_{s}=32.30 \mathrm{~ns}$ and $f_{c}=40 \mathrm{MHz}$. The optimization at each stage was carried out by 50 iterations in reconstruction with progressively increasing truncation numbers $(0,0),( \pm \mathrm{P} / 3, \pm \mathrm{Q} / 3),( \pm 2 \mathrm{P} / 3, \pm 2 \mathrm{Q} / 3)$ and $( \pm \mathrm{P}$, $\pm \mathrm{Q}$ ). Thus, total iteration number at each stage is 200 iterations. The original profile of the tunnel-like object and reconstructed results by IMS-FBTS at each stage are shown in Fig. 2. As can be seen, the IMS strategy yields a significant improvement. It is confirmed that IMS-FBTS 
method efficiently and accurately provides the reconstruction of cross borehole radar.

\section{CONCLUSIONS}

A novel methodology for cross-borehole imaging has been presented. The multiscaling procedure, exploiting the limited amount of information achievable from the scattering measurements, is integrated with the time domain inversion algorithm. In order to check the effectiveness of the proposed approach, some preliminary numerical results for a high-contrast object in a large investigation domain have been shown. Although a two-dimensional TM case has been considered, the proposed inversion algorithm can be easily extended to TE and three-dimensional cases.

\section{REFERENCES}

[1] G. A. Ellis and I. C. Peden, "Cross-borehole sensing: Identification and localization of underground tunnels in the presence of a horizontal stratification," IEEE Trans. Geosci. Remote Sens., vol. 35, pp. 756-761, May 1997.

[2] H. K. Choi and J. W. Ra, "Detection and identification of tunnel by iterative inversion from cross-borehole CW measurements,” Microwave Opt. Technol. Lett., vol. 21, pp. 458465, 1999.

[3] T. Miwa, M. Sato, and H. Niitsuma, "Subsurface fracture measurement with polarimetric borehole radar," IEEE Trans. Geosci. Remote Sens., vol. 37, no. 2, pp. 828-837, Mar. 1999.

[4] T. Takenaka, H. Jia, and T. Tanaka, "Microwave imaging of

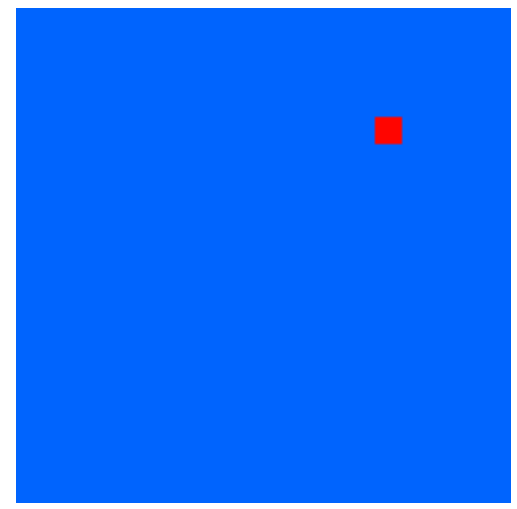

(a)

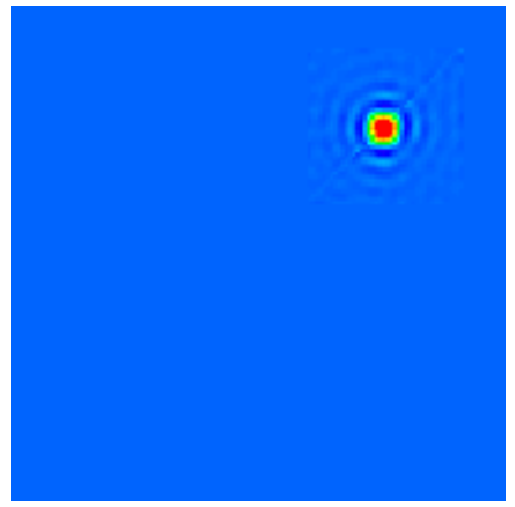

(c)

Fig. 2. Reconstructed relative permittivity. (a) Original profile. (b) Reconstruction after 200 iterations at 0th step of IMSA $(P, Q=67)$. (c) Reconstruction after 200 iterations at 1 st step of IMSA $(P, Q=22)$. (d) Reconstruction after 200 iterations at 2nd step of IMSA $(P, Q=5)$.

electrical property distribution by a forward-backward timestepping method,” J. Electromagn. Waves and Appl., vol. 14, no. 12, pp. 1609-1626, Dec. 2000. Geosci. Remote Sens., vol. 40, no. 7, pp. 1640-1647, Jul. 2002.

[6] S. Caorsi, M. Donelli, D. Franceschini, and A. Massa, “A new methodology based on a iterative multiscaling for microwave imaging,” IEEE Trans. Microwave Theory Tech., vol. 51, no. 4, pp. 1162-1173, Apr. 2003. multiscaling mehtod,” IEEE Trans. Microwave Theory Tech., vol. 52, no. 4, pp. 1217-1228, Apr. 2004.

[8] M. Donelli, G. Franceschini, A. Martini and A. Massa, "An integrated multiscaling strategy based on a particle swarm algorithm for inverse scattering problems,” IEEE Trans. Geosci. Remote Sens., vol. 44, no. 2, pp. 298-312, Feb. 2006.

[9] O. M. Bucci and T. Isernia, "Electromagnetic inverse scattering: Retrievable information and measurement strategies," Radio Sci., vol. 32, pp. 2123-2138, 1997.

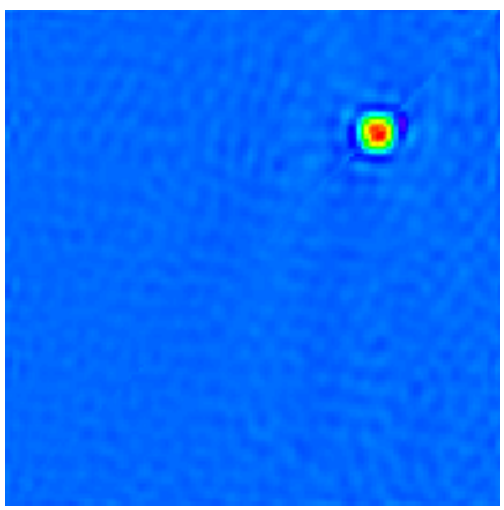

(b)

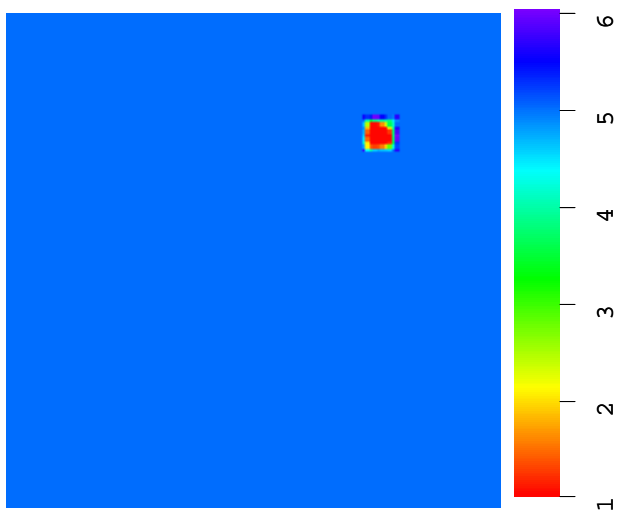

[5] H. Jia, T. Takenaka, and T. Tanaka, "Time-domain inverse scattering method for cross-borehole radar imaging,” IEEE Trans.

[7] S. Caorsi, M. Donelli, and A. Massa, "Detection, location, and imaging of multiple scatterers by means of the iterative 